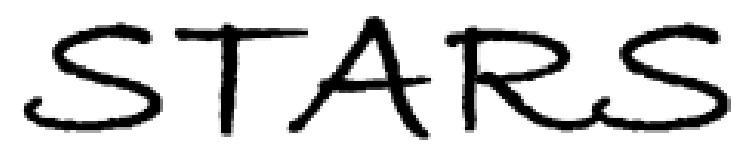

University of Central Florida

STARS

$1-1-2010$

\title{
Change detection of land use and land cover in an urban region with SPOT-5 images and partial Lanczos extreme learning machine
}

\author{
Ni-Bin Chang \\ University of Central Florida \\ Min Han \\ Wei Yao \\ Liang-Chien Chen \\ Shiguo Xu
}

Find similar works at: https://stars.library.ucf.edu/facultybib2010

University of Central Florida Libraries http://library.ucf.edu

This Article is brought to you for free and open access by the Faculty Bibliography at STARS. It has been accepted for inclusion in Faculty Bibliography 2010 s by an authorized administrator of STARS. For more information, please contactSTARS@ucf.edu.

\section{Recommended Citation}

Chang, Ni-Bin; Han, Min; Yao, Wei; Chen, Liang-Chien; and Xu, Shiguo, "Change detection of land use and land cover in an urban region with SPOT-5 images and partial Lanczos extreme learning machine" (2010). Faculty Bibliography 2010s. 4.

https://stars.library.ucf.edu/facultybib2010/4

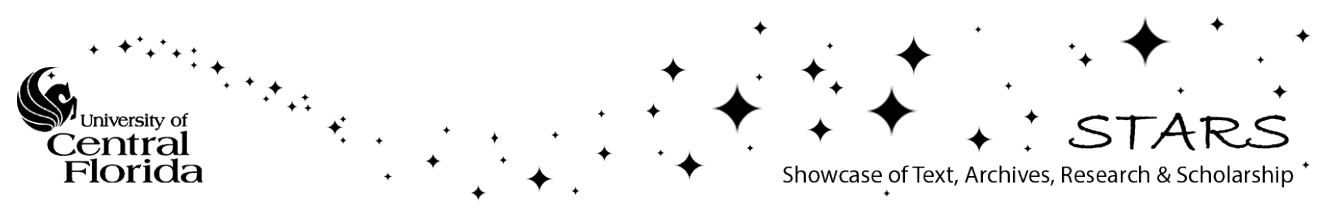




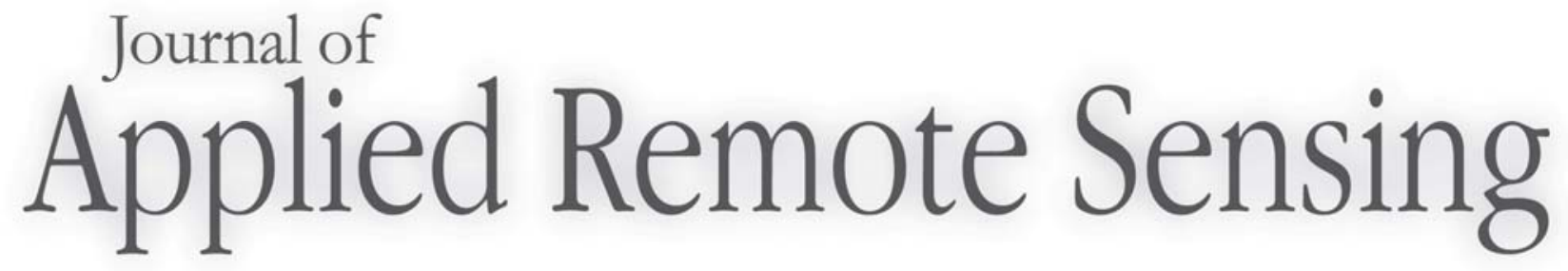

\section{Change detection of land use and land cover in an urban region with SPOT-5 images and partial Lanczos extreme learning machine}

Ni-Bin Chang

Min Han

Wei Yao

Liang-Chien Chen

Shiguo $\mathrm{Xu}$

\section{O SPIE}




\title{
Change detection of land use and land cover in an urban region with SPOT-5 images and partial Lanczos extreme learning machine
}

\author{
Ni-Bin Chang ${ }^{a}$, Min Han ${ }^{b}$, Wei Yao ${ }^{b}$, Liang-Chien Chen, ${ }^{c}$ \\ and Shiguo $\mathrm{Xu}^{\mathrm{d}}$ \\ ${ }^{\text {a }}$ University of Central Florida, Department of Civil and Environmental and Construction \\ Engineering, 4000 Central Florida Boulevard, Orlando, Florida 32816 USA \\ nchang@mail.ucf.edu \\ ${ }^{\mathrm{b}}$ Dalian University of Technology, College of Electronic and Information, No. 2 Linggong \\ Road, Ganjingzi District, Dalian City, Liaoning Province 116024 China \\ minhan@dlut.edu.cn, modestyao@mail.dlut.edu.cn \\ ${ }^{c}$ National Central University, Center for Space and Remote Sensing Research, No. 300, \\ Jhongda Road, Jhongli City, Taoyuan 32001 Taiwan \\ lcchen@csrsr.ncu.edu.tw \\ ${ }^{\mathrm{d}}$ Dalian University of Technology, School of Civil and Hydraulic Engineering, Institute of \\ Water and Environment Research, No. 2 Linggong Road, Ganjingzi District, \\ Dalian City, Liaoning Province 116024 China \\ sgxu@dlut.edu.cn
}

\begin{abstract}
Satellite remote sensing technology and the science associated with evaluation of land use and land cover (LULC) in an urban region makes use of the wide range images and algorithms. Improved land management capacity is critically dependent on real-time or near real-time monitoring of land-use/land cover change (LUCC) to the extent to which solutions to a whole host of urban/rural interface development issues may be well managed promptly. Yet previous processing with LULC methods is often time-consuming, laborious, and tedious making the outputs unavailable within the required time window. This paper presents a new image classification approach based on a novel neural computing technique that is applied to identify the LULC patterns in a fast growing urban region with the aid of 2.5-meter resolution SPOT-5 image products. The classifier was constructed based on the partial Lanczos extreme learning machine (PL-ELM), which is a novel machine learning algorithm with fast learning speed and outstanding generalization performance. Since some different classes of LULC may be linked with similar spectral characteristics, texture features and vegetation indexes were extracted and included during the classification process to enhance the discernability. A validation procedure based on ground truth data and comparisons with some classic classifiers prove the credibility of the proposed PL-ELM classification approach in terms of the classification accuracy as well as the processing speed. A case study in Dalian Development Area (DDA) with the aid of the SPOT-5 satellite images collected in the year of 2003 and 2007 and PL-ELM fully supports the monitoring needs and aids in the rapid change detection with respect to both urban expansion and coastal land reclamations.
\end{abstract}

Keywords: land use and land cover, computational intelligence, image processing, SPOT-5, PL-ELM

\section{INTRODUCTION}

Urbanization is the physical growth of urban areas [1]. Urbanization and associated land-use/land cover change (LUCC) is normally driven by a combination of socio-economic development and political considerations. In turn, urbanization and LUCC may end up numerous direct and indirect impacts on micro- and macro-level 
climatology, hydrology, and environmental ecology. Some of the LUCC impacts, such as pollution, land reclamation, and deforest, are even quite destructive. The ultimate impact of urbanization will eventually come back and affect urban dwellers as a result of feedbacks of climate change constrained by the varying hydrological cycle. Offering compelling and encompassing understanding of the causes and consequences of urbanization-related LUCC requires using new remote sensing satellite technologies.

Early studies of remote sensing image classification counted on statistical methods, such as the maximum likelihood (ML) classifier [2], the K-nearest neighbor (Knn) algorithm [3], and the K-means clustering approach [4]. In recent years, methods based on artificial intelligence and machine learning techniques have been popular in this field. Various classification approaches based on neural computing, fuzzy logic, evolutionary algorithm, and expert system are widely reported in the literature [5]-[11]. Neural computing methods are date-driven methods that have high fault-tolerance in general [5]-[7]. There are many successful application of remote sensing image classification using classifiers based on all kinds of different neural networks, such as back propagation (BP), radial basis function (RBF), self organized mapping (SOM) [12]-[14] and support vector machine (SVM) [9]. However, these neural networks are all "black box" models, whose classification mechanisms are hardly to be interpreted. Furthermore, problems such as over fitting, local minimum, and slow convergence speed are quite common for neural computing methods. Yet classifiers based on fuzzy logic are much easier to be interpreted because the classification is usually implemented according to some rules which are summarized from the training data set. Most fuzzy logic methods are hybrid methods, for example, the fuzzy c-means (FCM) algorithm [15] is a hybrid between fuzzy logic and the statistical algorithm (e.g., c-means). Classifiers based on fuzzy neural network (FNN) [10] and fuzzy Artmap [16], which are both combinations between fuzzy logic and neural networks, were reported too. Evolutionary algorithms are another category of machine learning techniques which are widely used in remote sensing image classification. Genetic algorithm (GA), evolutionary programming (EP), and genetic programming (GP) are some classical evolutionary algorithms which have many successful applications [17]-[19]. Classifier based on artificial immune system (AIS) [20] and swarm intelligence [21] can also be included in this category. In addition, classifiers such as those based on expert system theory [8] and decision tree techniques [22] are also representative and important classification methods.

The city of Dalian in China has been a fast growing region economically and socially as evidenced by the rapid expansion of the Dalian Development Area (DDA) in the past decade. Using remote sensing satellite images for monitoring the process-oriented movement of the urban sprawl and predicting human influences on landscapes and ecosystems becomes essential nowadays. The spatial and temporal patterns of LULC that shapes and transforms cities, suburbs, and rural area can be identified to at least understand the interaction among urbanization, economic development, land use policy, urban planning, and natural resources in this region. With the aid of modern remote sensing technologies, essential information about the urbanization process that simultaneously converge and diverge a fast growing urban region in many ways may be gained to answer several significant science questions in the future, including: 1) how urbanization alters the characteristics of natural disturbances? 2) how disturbance regimes are altered through human influences along urban/rural gradients? And 3) how the spatiotemporal aspects and management models of LUCC may help identify subsequent risks to human and natural environments during fast economic development? Such an endeavor leads to the possible linkages between human dimensions aspects of LUCC along urban/rural interfaces.

In our study, two scenes of SPOT-5 images with 2.5-meter resolution were acquired and classified to capture the spatiotemporal LULC patterns from 2003 to 2007 to inform policy makers, natural resource professionals, and private citizens regarding possible changes to ecosystem goods and services along urban/rural gradients. To tackle the spatial 
and temporal heterogeneity of LULC patterns, advanced algorithms for achieving reliable classification are required. Early studies of remote sensing image classification counted on statistical methods, such as the ML classifier. In recent years, methods based on artificial intelligence and machine learning techniques have been popular in this field. It is the aim of this paper to present a new classification approach based on a neural computing technique -- partial Lanczos bidiagonalization extreme learning machine (PL-ELM) [23,24], which provides better performance with extremely fast learning speed, as compared to classical neural computing techniques, such as backward propagation neural network (BP).

Table 1: Summary of classification methods in image processing

\begin{tabular}{|c|l|l|}
\hline \multicolumn{2}{|c|}{ Statistical Methods } & ML [2], Knn [3], K-means [4] \\
\hline \multirow{2}{*}{$\begin{array}{c}\text { Artificial } \\
\text { Intelligence }\end{array}$} & Neural Networks & BP [5,12,17], RBF [6], SOM [7], SVM [9] \\
\cline { 2 - 3 } & Fuzzy Logic & FCM [8,15], Fuzzy Artmap [9,16], FNN [10] \\
\cline { 2 - 3 } & Evolutionary Algorithms & GA [10,17], EP [11], GP [18], AIS [13], SI [14] \\
\hline \multicolumn{2}{|c|}{ Other Methods } & Expert system [8], Decision tree [15] \\
\hline
\end{tabular}

\section{DATA PREPARATION}

\subsection{High resolution SPOT-5 image products}

Due to technical limitations, high spatial resolution and large swath width of remote sensing images can hardly be achieved at the same time. For rural areas, high spatial resolution is literally not necessary and the large swath width will bring much more convenience. In the remote sensing of urban areas, however, higher spatial resolution is preferable, for the objects of our interest in the study of urban areas are usually with a smaller scale and can hardly be recognized in low resolution images. Furthermore, the swath widths of high resolution images are big enough for most urban areas.

Two scenes of SPOT-5/HRG images were collected for the research. These images were originally acquired during the overpasses of the SPOT- 5 satellite on May $5^{\text {th }}, 2003$ and April 13, 2007, respectively. The SPOT-5 Images used in our study cover an area larger than $39 \mathrm{~km} \times 40 \mathrm{~km}$, while the region we concerned about can be thoroughly included in a square area of $20 \mathrm{~km} \times 20 \mathrm{~km}$. Therefore, we cut subsets from the whole images in advance. The resolution of the original SPOT-5/HRG images in the green, red and near infrared channels is $10 \mathrm{~m}$, and the $2.5 \mathrm{~m}$ images we used are resolution enhanced image fusion products. The original SPOT-5/HRG images are actually in false color composite, and the true color composite images shown in Figure 1 are also image fusion products, in which the blue channel images are synthesized from the original SPOT-5/HRG images in the green, red and near infrared channels.

\subsection{Field campaigns for ground truthing}

The study area is a state run industrial complex - the DAA (i.e., about $121^{\circ} \mathrm{E}, 39^{\circ} \mathrm{N}$ ) administered by the city of Dalian. The DDA is located at the northeast of the city of Dalian. As illustrated in Figure 1, the DDA is right next to the seashore with the coast line of about $130 \mathrm{~km}$. To the north of the urban area is the Big Black Mountain. With a visual contact at the two remote sensing images in Figure 1, some obvious changes of LULC can be observed. Through the years from 2003 to 2007, large scale land reclamations along the shoreline happened in this area, which is of the major concern in this study. Quantitative information about these changes may be retrieved with the aid of the 
proposed classification method.

Six aggregated classes of land cover of interest in this study include buildings, roads, bare fields, grasslands, forests and water bodies that were categorized in this study eventually. Ground truth data was collected during the two field campaigns. 71 ground truth data sets are illustrated in Figure 2. The ground truth data base consisting of over 20,000 pixels were constructed in advance to generate both the training and the testing datasets for the classifiers. These ground truth samples cover all classes of LULC of interest. The differences within and between some classes of LULC were carefully considered during the sampling processes of the ground truth data, e.g., a variety of buildings were classified as the same type of LULC.

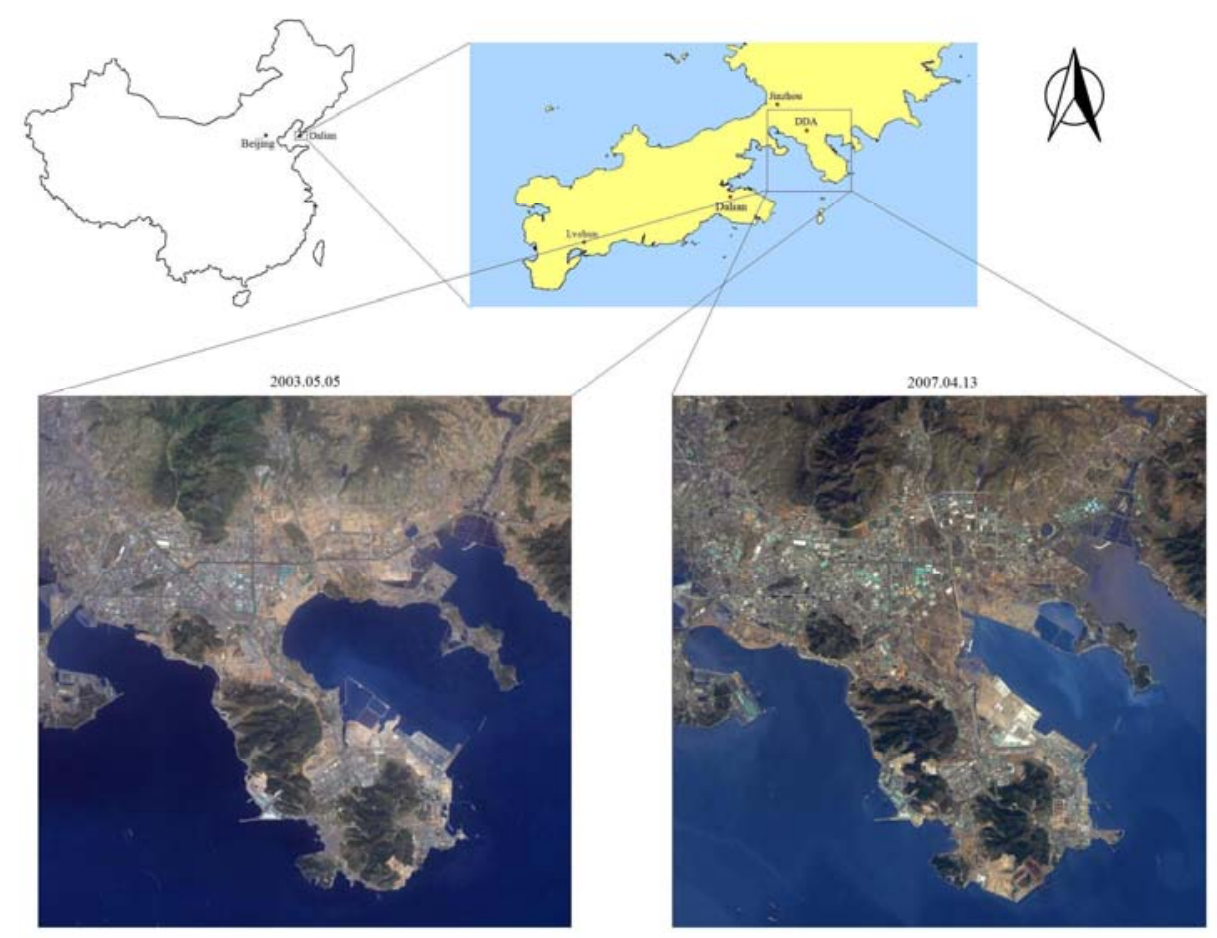

Figure 1. The location of the study area and the corresponding SPOT-5 images in 2003 and 2007.

\section{MATERIALS AND METHODS}

\subsection{Classification Method - the PL-ELM}

The classifier used in our study is based on the PL-ELM algorithm. PL-ELM is a mathematical development via an extreme learning machine (ELM), which is deemed a novel artificial intelligence algorithm for handling single hidden layer feedforward neural networks (SLFNs). Figure 3 demonstrates an example of a SLFN from which ELM can be honored for its great generalization performance and fast learning speed. It can be used to construct both regressors and classifiers in applications. Successful applications of ELM in different areas were reported frequently in literatures [25]-[28]. As an enhancement of ELM, PL-ELM provides even better performance and shows great potential to be the core algorithm as an effective and efficient classifier. 


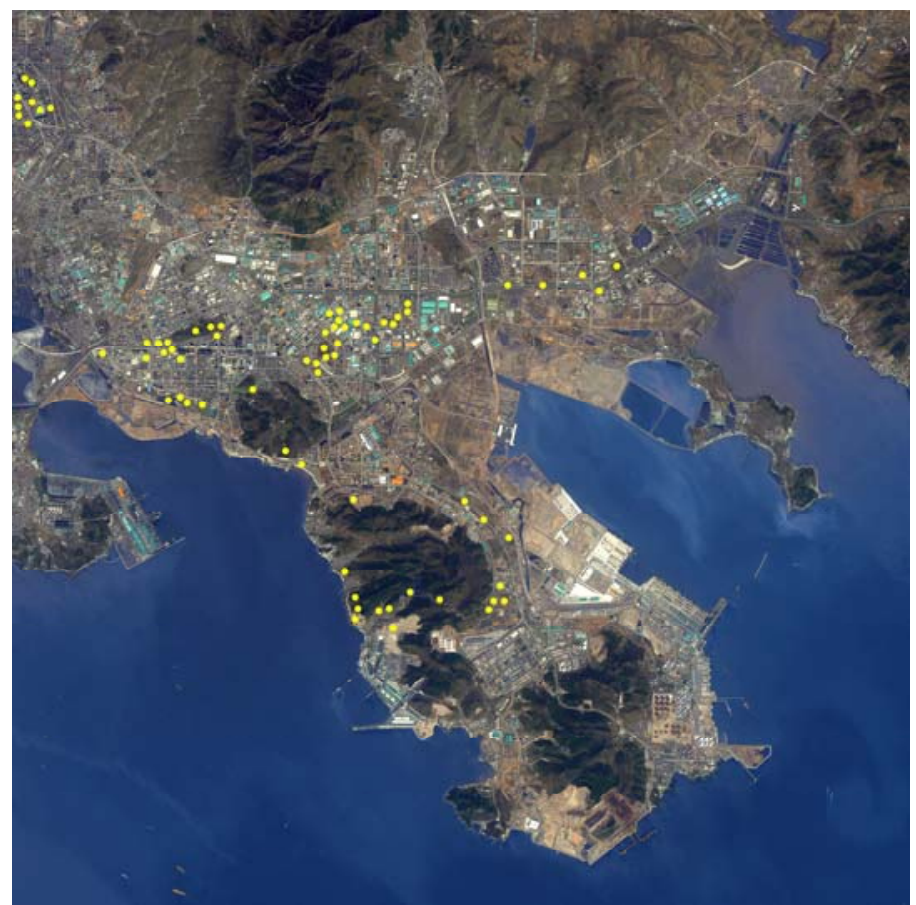

Figure 2. Distribution of ground truth data points in the study area.

In Figure 3, assume that there are $N$ training samples $\left(\mathbf{x}_{\mathbf{i}}, \mathbf{t}_{\mathbf{i}}\right) \in R_{n} \times R_{m}(\mathrm{i}=1,2, \ldots, N)$ for the construction of the classifier to establish a $n$-input $m$-output network. Within the context of an image classification application, $n$ and $m$ correspond to the numbers of pixel features and the classes of LULC of interest, respectively. $\boldsymbol{a}_{\boldsymbol{i}}=\left[a_{i 1}, a_{i 2}, \ldots, a_{i n}\right]^{T}(i=1,2, \ldots, L)$ is the weight vector connecting the $i$ th hidden node with all the input nodes, and $v_{\boldsymbol{i}}=\left[v_{i 1}, v_{i 2}, \ldots, v_{i m}\right]^{T}(i=1,2, \ldots, L)$ is the weight vector connecting the $i$ th hidden node and the output nodes. $b_{i}$ is the bias of the ith hidden node.

The forward calculation process of the network to produce the prediction $\boldsymbol{Y}$ can be expressed as

$$
\boldsymbol{H} \boldsymbol{V}=\boldsymbol{Y}
$$

where

$$
\boldsymbol{V}=\left[\begin{array}{l}
\boldsymbol{v}_{1}^{T} \\
\vdots \\
\boldsymbol{v}_{L}^{T}
\end{array}\right]_{L \times m}, \quad \boldsymbol{Y}=\left[\begin{array}{l}
\mathbf{y}_{1}^{T} \\
\vdots \\
\mathbf{y}_{N}{ }^{T}
\end{array}\right]_{N \times m}
$$

$\boldsymbol{H}$ is called the hidden layer output matrix of the neural network, and it can be calculated following

$$
\boldsymbol{H}=\left[\begin{array}{cccc}
g\left(\boldsymbol{a}_{1}, \boldsymbol{b}_{1}, \mathbf{x}_{1}\right) & g\left(\boldsymbol{a}_{2}, \boldsymbol{b}_{2}, \mathbf{x}_{1}\right) & \ldots & g\left(\boldsymbol{a}_{L}, \boldsymbol{b}_{L}, \mathbf{x}_{1}\right) \\
\vdots & \vdots & & \vdots \\
g\left(\boldsymbol{a}_{1}, \boldsymbol{b}_{1}, \mathbf{x}_{N}\right) & g\left(\boldsymbol{a}_{2}, \boldsymbol{b}_{2}, \mathbf{x}_{N}\right) & \ldots & g\left(\boldsymbol{a}_{L}, \boldsymbol{b}_{L}, \mathbf{x}_{N}\right)
\end{array}\right]_{N \times L}
$$


The sigmoid function is employed as the active function for the hidden layer notes, therefore,

$$
g\left(a_{i}, b_{i}, \mathbf{x}_{i}\right)=\operatorname{sig}\left(a_{i} \mathbf{x}_{i}+b_{i}\right)
$$

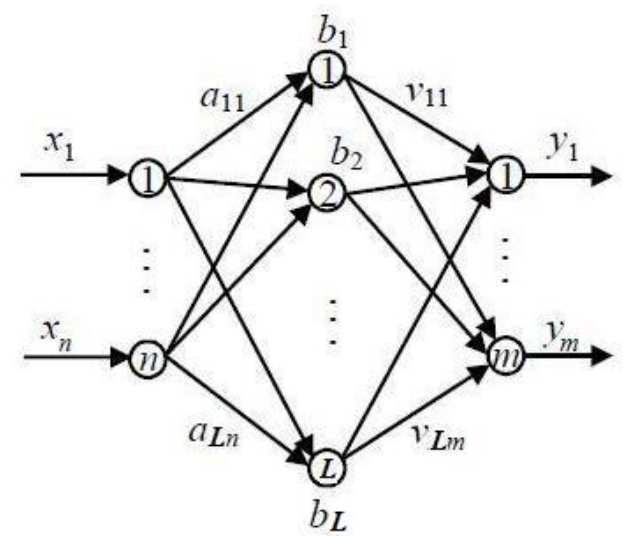

Figure 3. A single hidden layer feedforward neural network.

During the learning process, it can be proved that the input weights and hidden layer biases can be randomly chosen, and the hidden layer output matrix $H$ may remain unchanged [14]. Thus, the output weights are actually the only parameters of the SLFN to be tuned, and the training process is equivalent to finding the minimal norm solution for the linear system $\boldsymbol{H} \boldsymbol{V}=\boldsymbol{T}$, where $T$ is the target output matrix that

$$
\boldsymbol{T}=\left[\begin{array}{l}
\mathbf{t}_{1}{ }^{T} \\
\vdots \\
\mathbf{t}_{N}{ }^{T}
\end{array}\right]_{N \times m}
$$

The linear system can be solved using the least square method (LSM), such that

$$
\boldsymbol{V}=\boldsymbol{H}^{\dagger} \boldsymbol{T}
$$

Where $\boldsymbol{H}^{\dagger}$ denotes the Moore-Penrose generalized inverse [29] of $H$.

In ELM, the computing of the Moore-Penrose generalized inverse is based on the singular value decomposition (SVD) of $\boldsymbol{H}$. And the SVD of $\boldsymbol{H}$ is the major time consuming and the most essential process during the training of the SLFN. In PL-ELM, on the other hand, the output weights are calculated by the hybrid of partial Lanczos bidiagonalization [30] and SVD. As compared to ELM, PL-ELM can effectively filter out the adverse effects caused by those small singular values in the ill-conditioned hidden layer output matrix; furthermore, by implementing the partial SVD instead of the full one, the computational complexity can also be reduced [9].

\subsection{Classification based on multiple features}

The false color SPOT-5 images contain only three channels, and the synthesized blue channel in the true color images is merely a linear combination of the original green, red and near infrared channels. Theoretically speaking, data points corresponding to these 
image pixels are in a three-dimension feature space. Large amounts of data points in low level feature spaces tend to cluster making them more difficult to be distinguished and correctly classified.

In order to add more features to the pixels, we introduced the normalized differential vegetation index (NDVI) and four commonly used texture features including angular second moment, contrast, correlation, and homogeneity [31]. Therefore, the dimension of the feature space for all the data points/pixels is extended to eight.

The NDVI can be calculated from the red and near infrared channels of the SPOT-5 images as follows:

$$
N D V I=\frac{N I R-R E D}{N I R+R E D}
$$

where NIR and RED denote the digital numbers (DNs) of the near infrared channel and red channel images, respectively. As a matter of fact, the exact definition for NDVI is based on the spectral reflectance rather than the DNs. Hence, the equation (3) is a simplification for the calculation of NDVI. This simplification is not compatible for quantitative assessments, but it won’t bring unpleasant subsequences in a classification application.

During the classification procedures, it's not rare that different kind of LULC exhibits similar spectral patterns, especially for those images with low spectral resolutions (large spectral span and few spectral bands). However, these spectrally resembling objects can be distinguished by their different texture patterns. So texture features, usually as a complement to the spectral features, are functional in dealing with image classification in many cases [32]. In our study, the four kinds of texture features as mentioned beforehand are calculated from a $9 \times 9$ gray level co-occurrence matrix (GLCM) with respect to every pixel.

\section{EXPERIMENTS}

\subsection{Classification steps}

The proposed classification approach follows four major steps as below. First, additional features including NDVI and texture features are extracted from the original remote sensing images to construct multi-feature image sets. Second, training data sets and the testing data sets are defined and prepared based on the ground truth data. Third, the classifier is trained using the training data sets and evaluated using the testing data sets. Fourth, the full image sets are imported into the well-trained classifier to produce the final classification images of the study region. These four steps are illustrated as data/information flows and denote as step 1, 2, 3 and 4 in Figure 4.

The extraction of additional features described in detail previously supports both the training and testing data sets. Given that the type of LULC was divided into six major categories, namely water bodies, forests, grasslands, bare fields, buildings, and roads, multitemporal variations of grasslands due to urban sprawl, water coverage due to coastal land reclamation, and buildings due to population increase and migration would reflect the main direction and connection of the urbanization process, which are the major concern of our study.

The better the classifier trained, the higher the odds of success when the classifier encounters similar objects to discern and distinguish within the same categories. Major challenges are that remarkable similarity and varieties exist within some of these six LULC classes, and these disturbances may impact the final performance of the classifier. To deal with this issue, during the classification process, these six major classes of LULC were further divided into fourteen sub-classes of LULC. These sub-classes of urban LULC patterns are described and summarized in Table 2. 
Table 2. Basic LULC classes and sub classes.

\begin{tabular}{|l|l|l|}
\hline Basic LULC type & Sub type & Explanation \\
\hline \multirow{3}{*}{ Water body } & Water 1 & Clean water \\
\cline { 2 - 3 } & Water 2 & Turbid water \\
\hline \multirow{3}{*}{ Forest } & Forest 1 & Dark green trees \\
\cline { 2 - 3 } & Forest 2 & Yellow green trees \\
\cline { 2 - 3 } & Forest 3 & Sparse forest \\
\hline \multirow{3}{*}{ Grass land } & Grass 1 & Light green grass \\
\cline { 2 - 3 } & Grass 2 & Dark green grass \\
\hline Bare filed & Bare field & No subclasses \\
\hline \multirow{3}{*}{ Building } & Building 1 & Buildings in residential areas \\
\cline { 2 - 3 } & Building 2 & Factory buildings with blue roofs \\
\cline { 2 - 3 } & Building 3 & Factory buildings with orange roofs \\
\cline { 2 - 3 } & Building 4 & Factory buildings with gray roofs \\
\cline { 2 - 3 } & Building 5 & Factory buildings with white roofs \\
\hline Road & Road & No subclasses \\
\hline
\end{tabular}

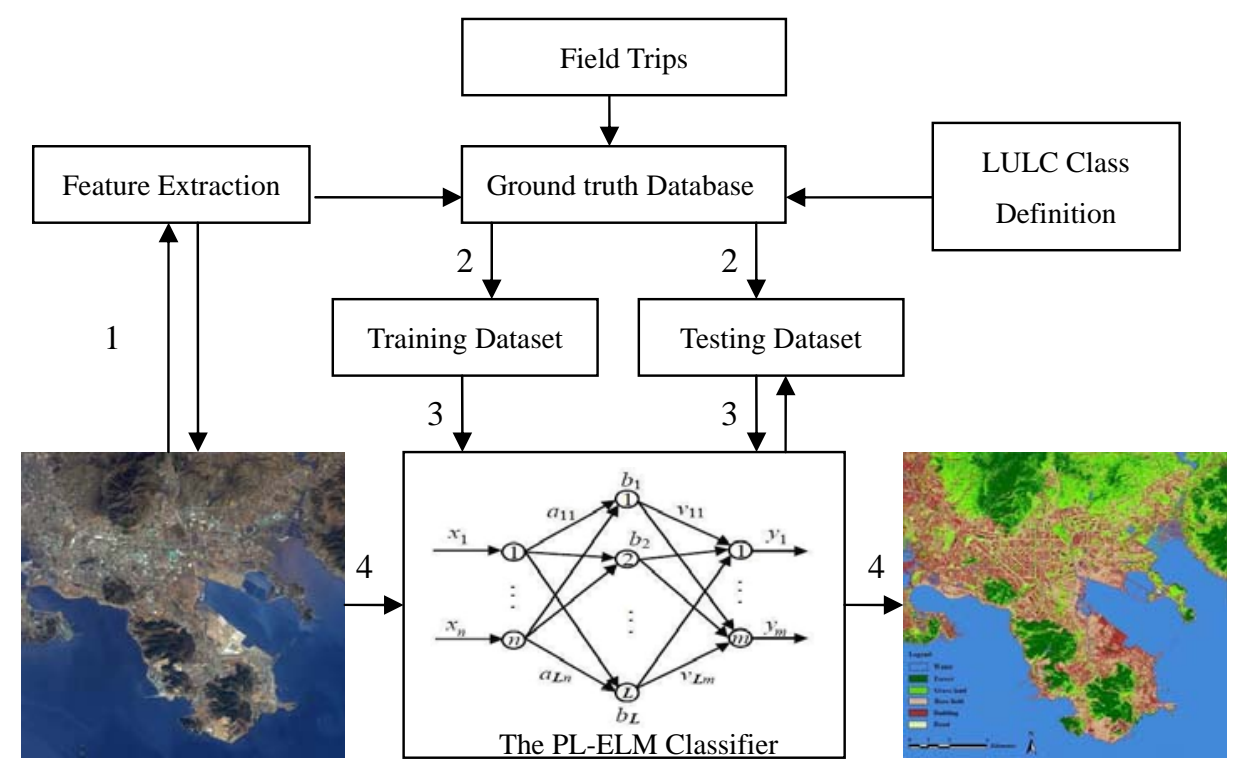

Major experimental steps:

1. Extract multiple features from the original remote sensing images;

2. Construct the training data set and testing data set based on the ground truth data base;

3. Train the classifier with the training data set, and test its performance with the testing data set;

4. Classifv the full scale image of the studv area using the PL-ELM classifier.

Figure 4. The flowchart of the proposed classification approach.

To improve the prediction accuracy, 500 data points from the ground truth data set associated with each sub-class were sampled to build the training data set, and another 500 associated with each sub-class were sampled to form the testing data set. The PL-ELM classifier can then be trained using the training data set and the performance of the proposed classification approach may be evaluated further by using the testing data set. Comparisons with other classifiers could clarify the question as to "how good the PL-ELM classifier could be, comparatively speaking?” Once the credibility of the 
PL-ELM classifier can be confirmed after the comparisons among all applicable classifiers can be completed, the best trained classifier would be applicable to carry out the implementation in the study area. Once the task can be completed, direct outputs of the classifier, which are expressed as 14 sub-classes, may be reorganized into the six major classes of LULC for the final assessment of classification accuracy and final presentation of all thematic maps accordingly.

The accuracy of classification was assessed with regard to the overall accuracy (OA), user's accuracy (UA), and producer's accuracy (PA) [33] at first. The ultimate accuracy of classification may be carried out by using the Kappa statistics [34] based on the confusion matrix. The Kappa coefficient is defined based on the confusion matrix as follows:

$$
\text { Kарра }=\frac{N \sum_{k=1}^{r} x_{k k}-\sum_{k=1}^{r}\left(x_{k+} \times x_{+k}\right)}{N^{2}-\sum_{k=1}^{r}\left(x_{k+} \times x_{+k}\right)}
$$

where $r$ is the number of rows in the confusion matrix, $x_{k k}$ is the number of observations in row $i$ and column $j, x_{k+}$ and $x_{+k}$ are the marginal totals for row $i$ and column $j$, respectively, and $N$ is the total number of observations.

\subsection{Classification outcome}

As reported in Table 3, these statistics were calculated based on the testing data set of 2007 for the purpose of demonstration. Predictions generated by the PL-ELM classifier, which are design to sort out fourteen sub-classes of LULC. Then aggregation of land use sub-classes into major categories can be carried out by following the hierarchical order in Table 1. The performance of the PL-ELM classifier can be grossly compared with some other representative supervised classifiers, such as BP, ML, Knn, Naïve Bayes (NB) and SVM, using the same training and testing data sets. Within these classifiers selected, BP is among the most classic neural computing technique [35]. With the aid of the PL-ELM classifier, the BP classifier was fully implemented via a SLFN. ML and Knn, however, are two powerful traditional classification methods [36]. NB is a probabilistic method based on the Bayesian theorem, which can often outperform more sophisticated classification methods despite its simplicity [37]. SVM is a very popular state-of-the-art machine learning algorithm [38]. The classification accuracies (PA) for each major type of LULC were then compared with one another leading to the generation of the overall accuracies and the Kappa coefficients. As shown in Table 4, the PL-ELM classifier achieves the best overall accuracy and the Kappa coefficient based on the testing data set. When looking into the detail, the PL-ELM classifier obviously outperforms the BP, ML, NB and SVM classifiers, while shows almost equivalent ability as the Knn classifier.

Table 3. Evaluation of the PL-ELM classifier

\begin{tabular}{|c|c|c|c|c|c|c|c|c|}
\hline & & \multicolumn{6}{|c|}{ Actual conditions } & \\
\hline & & $\begin{array}{l}\text { Water } \\
\text { body }\end{array}$ & Forest & $\begin{array}{c}\text { Grass } \\
\text { land }\end{array}$ & $\begin{array}{l}\text { Bare } \\
\text { field }\end{array}$ & Building & Road & $\begin{array}{l}\text { PA } \\
(\%) \\
\end{array}$ \\
\hline \multirow{7}{*}{ Predictions } & Water body & 997 & 1 & 0 & 0 & 2 & 0 & 99.7 \\
\hline & Forest & 0 & 1386 & 100 & 1 & 12 & 1 & 91.4 \\
\hline & Grass land & 0 & 77 & 921 & 0 & 2 & 0 & 92.1 \\
\hline & Bare field & 0 & 0 & 19 & 408 & 59 & 14 & 81.6 \\
\hline & Building & 11 & 14 & 10 & 34 & 2311 & 120 & 92.4 \\
\hline & Road & 0 & 0 & 1 & 8 & 71 & 420 & 84 \\
\hline & UA(\%) & 98.9 & 93.8 & 87.6 & 90.5 & 93.3 & 75.7 & \\
\hline \multicolumn{2}{|c|}{$\mathrm{OA}(\%)$} & \multicolumn{7}{|c|}{92.0} \\
\hline \multicolumn{2}{|c|}{ Kappa coefficient } & \multicolumn{7}{|c|}{0.8976} \\
\hline
\end{tabular}

Note: OA - overall accuracy; UA - user's accuracy; PA - producer's accuracy 
The image of a sub-region of the study area is segmented out from the 2007 SPOT- 5 image for the purpose of illustration in Figure 5. This sub-region image corresponds to the neighborhood of a small campus. The image is classified using the BP classifier, the ML classifier, the Knn classifier, the PL-ELM classifier, respectively. The classification results in Figure 5 may reveal a comparative analysis visually. The numbered circles on these LULC maps highlight the places where the PL-ELM classification shows relatively better quality. For example, the roads in circle 1 were misidentified as grassland and building using the BP classifier, whereas they were correctly classified by the PL-ELM classification approach.

Comparisons of processing speeds between the PL-ELM classifier and other classifiers were also made possible in terms of training and testing time period. The PL-ELM, BP, ML, Knn, NB,and SVM classifiers can be all implemented in MATLAB [39]. Such experiments associated with these selected classifiers were carried out under the environment of Windows XP with Pentium E2180 2.00GHz Dual CPU and 2.00GHz DDR800 RAM. All of them are comparable in these speed campaigns.

Table 4. Comparisons of classification accuracies with respect to different classifiers

\begin{tabular}{|l|c|c|c|c|c|c|}
\hline & PL-ELM & BP & ML & Knn & NB & SVM \\
\hline Water body & 99.7 & 99.5 & 98 & 99.4 & 98.9 & 77.5 \\
\hline Forest & 91.4 & 91.4 & 90.8 & 93.9 & 85.2 & 77.1 \\
\hline Grass land & 92.1 & 67.6 & 85.6 & 91.1 & 88.4 & 78 \\
\hline Bare field & 81.6 & 77 & 75 & 83.6 & 71.6 & 77.4 \\
\hline Building & 92.4 & 86 & 87.2 & 90.1 & 82.4 & 99.9 \\
\hline Road & 84 & 39.4 & 74.6 & 83.8 & 64.0 & 78.8 \\
\hline OA (\%) & 92.0 & 82.5 & 87.5 & 91.5 & 84.1 & 85.6 \\
\hline Kappa & 0.8976 & 0.7733 & 0.8398 & 0.8905 & 0.7972 & 0.8067 \\
\hline
\end{tabular}

Note: Backward Prorogation Neural Network (BP), Maximum likelihood (ML), K-nearest neighbor (Knn), Naïve Bayes (NB) and Support Vector Machine (SVM)

Using the training and testing data sets with 500 data points for each sub-classes of LULC, the training and testing time required for all classifiers were recorded and reported in Table 5. When compared with one another, the BP classifier worked by a sloppy way during both the training and the testing processes. The training speed of the PL-ELM was faster than the training speed of ML. But the testing speed of the PL-ELM cannot outperform the testing speed of ML. Nevertheless, the processing speed of the PL-ELM and the ML were basically at the same level. The training process of the Knn classifier was extremely faster than the others. Yet the testing time period of the Knn took ten times longer than the testing time period of the PL-ELM and the ML. The testing time period implies actual time needed for the prediction of that classifier, thereby making the Knn out of our choice for a large-scale high resolution remote sensing image processing. The SVM suffers from the same problem as the Knn. The PL-ELM and the ML performed almost equally well though. The NB shows fairly high speed, though its prediction precision is not comparable with the PL-ELM and the ML, as reported in Table 2.

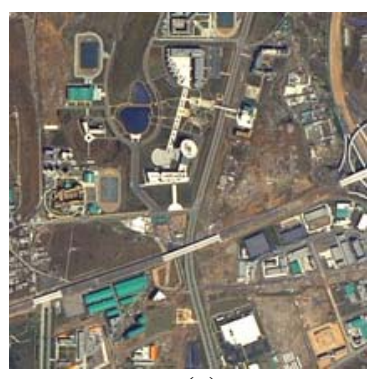

(a)

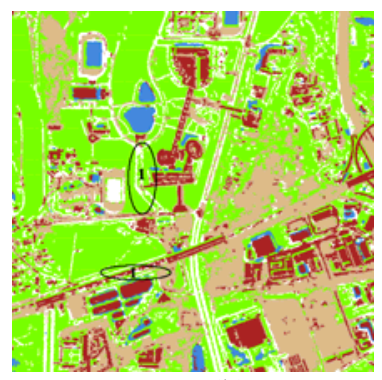

(b)

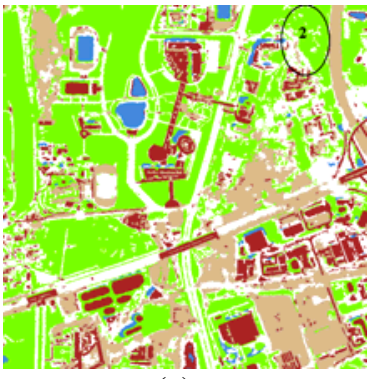

(c) 


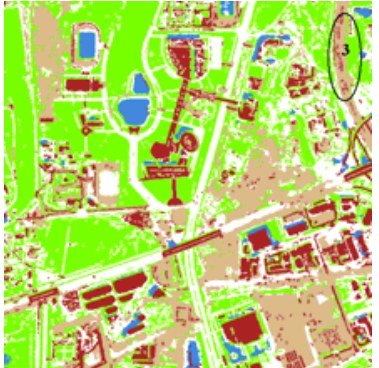

(d)

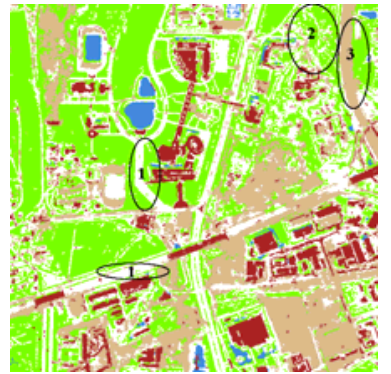

(e)

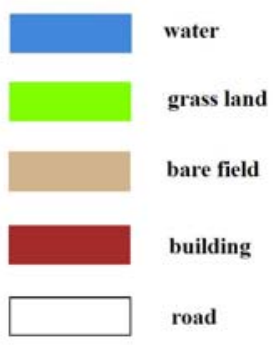

road

Figure 5. (a) The original image of a sub region and classification results produced using (b) the BP classifier, (c) the ML classifier, (d) the Knn classifier, (e) the PL-ELM classifier, respectively.

Figure 5 shows the classification results illustrating the LULC conditions of DDA in the year of 2003 and 2007, respectively, using the PL-ELM classifier. Table 6 summarizes the LUCC over the five years. These statistics are derived from the classification results with respect to the LUCC across the whole study area as illustrated in Fig. 6. The land reclamation and urban expansion processes can be verified by these statistics, while such LUCC can even be easily captured based on visual comparisons. From 2003 to 2007, the area of water body had decreased by $12.7206 \mathrm{~km}^{2}$ as a result of land reclamations. On the other hand, the building coverage in the region had increased by $7.8086 \mathrm{~km}^{2}$, which is a strong evidence for the rapid urban expansion process. The statistics in the table also imply severe deforests happened during this time period.

The rapid increases of brown color area, which stands for buildings, indicate a rapid urbanization process due to population growth and migration during a four-year time period. The exorbitant decreases of water coverage due to the coastal land reclamation turn out to be a symbolic pattern of the urbanization process in DDA driven by the land management policy. In any circumstance, the reduction of grassland drastically during such a short-time period implies a loss of ecosystem service in this urban region. Impermeable pavement came with the urbanization resulting in a distorted hydrological cycle. The high rate of the coastal land reclamation that improves the connectivity of the coastal industrial complex is also a cause of concern of seawater quality and soil salinity at the same time. Yet the positive aspects of urbanization leading to regional economic development should not be overshadowed by deterioration in the physical environment and quality of life in the region as long as there is no widening gap between supply and demand for public infrastructures.

Table 5. Comparisons of processing speeds with different classifiers

\begin{tabular}{|c|c|c|c|c|c|c|}
\hline & PL-ELM & BP & ML & Knn & NB & SVM \\
\hline Training Time (s) & 1.295663 & 291.220258 & 1.728073 & 0.00157 & 0.030319 & 1.601440 \\
\hline Testing Time (s) & 0.576115 & 58.979165 & 0.397023 & 7.96890 & 0.044070 & 6.030638 \\
\hline Total Time (s) & 1.871778 & 350.199423 & 2.125096 & 7.97047 & 0.074389 & 7.632078 \\
\hline
\end{tabular}

Table 6. Statistics of the LUCC in the area from 2003 to 2007.

\begin{tabular}{|l|c|c|c|}
\hline Category & $2003\left(\mathrm{~km}^{2}\right)$ & $2007\left(\mathrm{~km}^{2}\right)$ & changes $\left(\mathrm{km}^{2}\right)$ \\
\hline Water body & 178.3317 & 165.6111 & -12.7206 \\
\hline Forest & 74.6523 & 54.2095 & -20.4428 \\
\hline Grass land & 45.7958 & 55.1116 & 9.3158 \\
\hline Bare field & 21.3317 & 36.3092 & 14.9775 \\
\hline Building & 59.4946 & 67.3032 & 7.8086 \\
\hline Road & 20.3939 & 21.4554 & 1.0615 \\
\hline
\end{tabular}




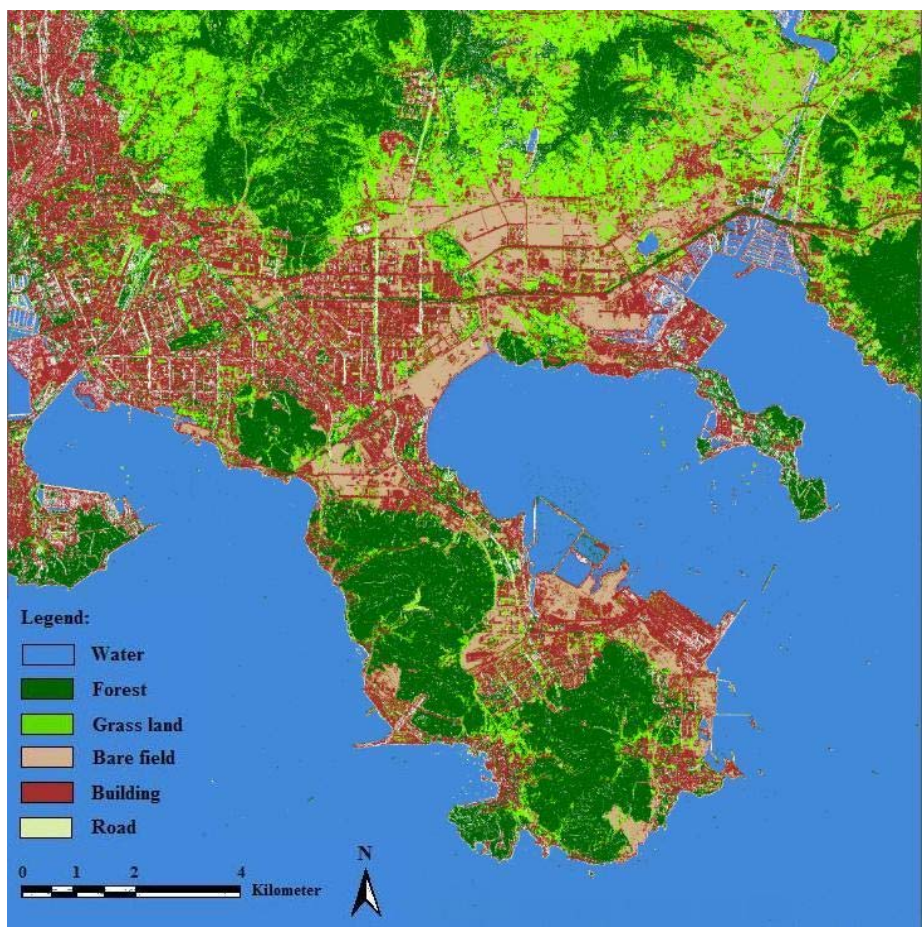

(a) 2003 LULC map

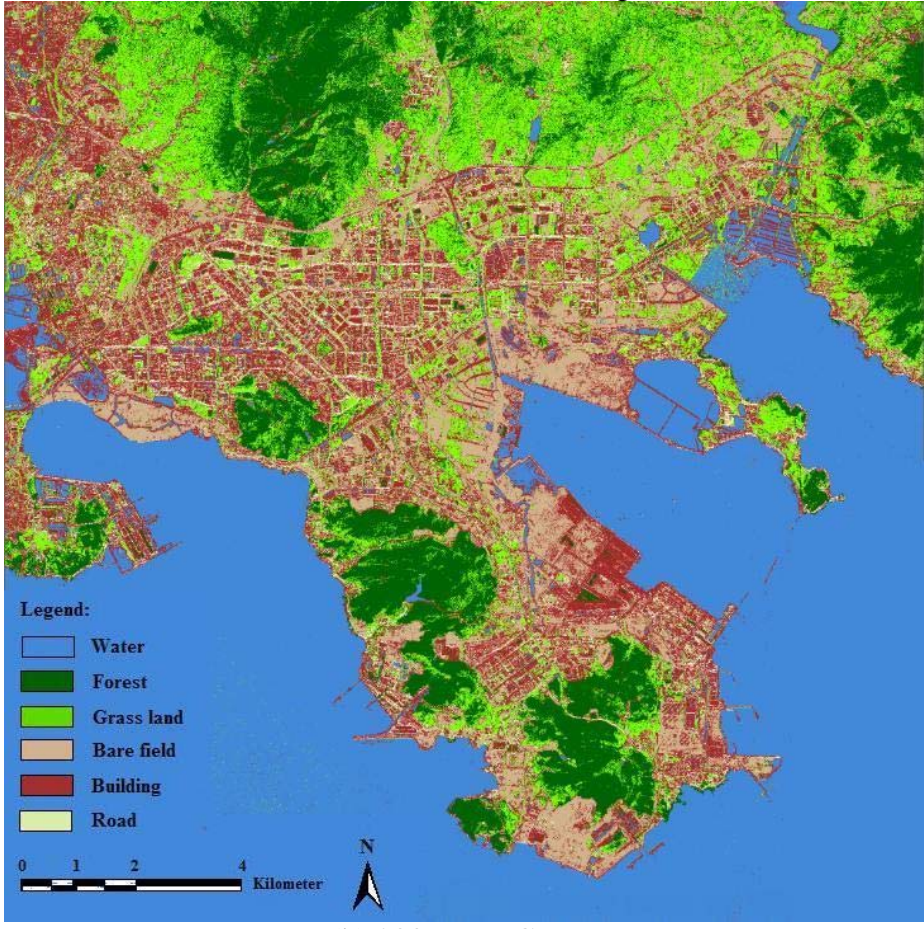

(b) 2007 LULC map

Figure 6. Final classification results produced from (a) the 2003 image and (b) the 2007 image using the PL-ELM classifier. 


\section{CONCLUSIONS}

Remote sensing plays an important role in urban environmental and hydrologic studies such as dynamic monitoring of urban sprawl and LUCC analyses. In our study, a novel LULC classification approach was developed based on an innovative machine learning algorithm, the PL-ELM. The proposed approach was applied to two SPOT-5 image sets acquired in 2003 and 2007, respectively, to produce six-category LULC maps with the resolution of $2.5 \mathrm{~m}$. To improve the prediction accuracy, additional features were extracted from the original remote sensing images and taken into account during the classification procedures. The classification accuracy of the proposed approach were assessed against the testing data set, which consists of 500 ground truth data points, and the performance of the PL-ELM classifier was fully verified in these computational experiments. Rapid urbanization processes, especially the coastal land reclamation process, can be recognized from the changes of the two LULC maps distinctively in the DDA. This advancement can be further applied to perform rapid change detection in urban region for land management, hazard mitigation, emergency response, and environmental management in the future.

\section{References}

[1] http://en.wikipedia.org/wiki/Urbanization

[2] P. Lombardo and C. J. Oliver, "Maximum likelihood approach to the detection of changes between multitemporal SAR images," IEEE Proc.:Radar, Sonar and Navigation, 148(4), 200-210 (2001) [doi: 10.1049/ip-rsn:20010114].

[3] E. Blanzieri and F. Melgani, "Nearest neighbor classification of remote sensing images with the maximal margin principle,” IEEE Trans. Geosci. Rem. Sens., 46(6), 1804-1811 (2008) [doi: 10.1109/TGRS.2008.2010682].

[4] J. Zhang, Z. Cui, A. Liu, and Y. Jia, "A k-means remote sensing image classification method based on adaboost." IEEE International Conference on Natural Computation, (ICNC '08). 4, 27 - 32 (2008) [doi: 10.1109/ICNC.2008.903].

[5] G. Giacinto, and F. Roli, "Design of effective neural network ensembles for image classification purposes,” Image Vision Comput, 19(9-10), 699-707, (2001). [doi: 10.1016/S0262-8856(01)00045-2].

[6] T. Kavzoglu, "Increasing the accuracy of neural network classification using refined training data,” Environ. Modell. Softw., 24(7), 850-858, (2009). [doi:10.1016/j.envsoft.2008.11.012].

[7] M. J. Canty, "Boosting a fast neural network for supervised land cover classification," Comput. Geosci., 35(6), 1280-1295, (2009). [doi: 10.1016/j.cageo.2008.07.004].

[8] W. L. Stefanov, M. S. Ramsey, and P. R. Christensen, "Monitoring urban land cover change: An expert system approach to land cover classification of semiarid to arid urban centers,” Remote Sens. Environ., 77(2), 173-185, (2001). [doi:10.1016/S0034-4257(01)00204-8].

[9] G. M. Foody, and A. Mathur, "Toward intelligent training of supervised image classifications: directing training data acquisition for SVM classification,” Remote Remote Sens. Environ., 93(1-2), 107-117, (2004). [doi:10.1016/j.rse.2004.06.017].

[10] H. W. Chen, N. B. Chang, R. F. Yu and Y. W. Huang, "Urban land use and land cover classification using the neural-fuzzy inference approach with Formosat-2 data," $J$. Appl. Remote Sens., 3, 033558-18, (2009). [doi:10.1117/1.3265995].

[11] B. C. K. Tso and P. M. Mather, "Classification of multisource remote sensing imagery using a genetic algorithm and Markov random fields,” IEEE Trans. Geosci. Remote Sens., 37(3), 1255-1260, (1999).[doi: 10.1109/36.763284].

[12]P. D. Heermann and N. Khazenie, "Classification of multispectral remote sensing data using a back-propagation neural network,” IEEE Trans. Geosci. Remote Sens., 
30(1), 81-88 (1992) [doi: 10.1109/36.124218].

[13] S. Suresh, N. Sundararajan and P. Saratchandran, "A sequential multi-category classifier using radial basis function networks,” Neurocomputing, 71(7-9), 1345-1358, (2008) [doi: 10.1016/j.neucom.2007.06.003].

[14]H. M. Chi and O. K. Ersoy, “A statistical self-organizing learning system for remote sensing classification,” IEEE Trans. Geosci. Remote Sens., 43(8), 1890-1900, (2005) [doi: 10.1109/TGRS.2005.851188].

[15] J. Fan, M. Han and J. Wang, "Single point iterative weighted fuzzy c-means clustering algorithm for remote sensing image segmentation,” Pattern Recognit., 42(11), 2527-2540, (2009) [doi:10.1016/j.patcog.2009.04.013].

[16] M. Han, X. Tang and L. Cheng, "An improved fuzzy ARTMAP network and its application in wetland classification." Geoscience and Remote Sensing Symposium (IGARSS '04) 5, 3432-3435, (2004) [doi: 10.1109/IGARSS.2004.1370443].

[17] M. Awad, K. Chehdi and A. Nasri, "Multicomponent image segmentation using a genetic algorithm and artificial neural network," IEEE Geosci. Remote Sens. Lett., 4(4), 571-575, (2007) [doi: 10.1109/LGRS.2007.903064].

[18]B. J. Ross, A. G. Gualtieri, F. Fueten, and P. Budkewitsch, "Hyperspectral image analysis using genetic programming,” Appl. Soft Comput., 5(2), 147-156 (2005) [doi: 10.1016/j.asoc.2004.06.003].

[19]D. Agnelli, A. Bollini and L. Lombardi, "Image classification: an evolutionary approach,” Pattern Recognit. Lett., 23(1-3), 303-309, (2002) [doi:10.1016/S0167-8655(01)00128-3].

[20] Y. Zhong, L. Zhang, J. Gong, and P. Li, “A supervised artificial immune classifier for remote-sensing imagery,” IEEE Trans. Geosci. Remote Sens., 45(12), 3957-3966, (2007) [doi: 2007ITGRS.45.3957Z].

[21] A. Daamouche and F. Melgani, "Swarm intelligence approach to wavelet design for hyperspectral image classification,” IEEE Geosci. Remote Sens. Lett., 6(4), 825-829, (2009) [doi: 10.1109/LGRS.2009.2026191].

[22] M. A. Friedl and C. E. Brodley, "Decision tree classification of land cover from remotely sensed data,” Remote Sens. Environ., 61(3), 399-409, (1997) [doi: 10.1016/S0034-4257(97)00049-7].

[23] X. Tang and M. Han, "Partial Lanczos extreme learning machine for single-output regression problems,” Neurocomputing, 72(13-15), 3066-3076, (2009). [doi: 10.1016/j.neucom.2009.03.016].

[24] G. Wang, Y. Zhao and D. Wang, “A protein secondary structure prediction framework based on the extreme learning machine,” Neurocomputing, 72(1-3), 262-268, (2008) [doi: 10.1016/j.neucom.2008.01.016].

[25] Z. Runxuan, G. B. Huang, N. Sundararajan and P. Saratchandran, "Multicategory classification using an extreme learning machine for microarray gene expression cancer diagnosis,” IEEE/ACM Trans. Comput. Biol. Bioinf., 4(3), 485-495, (2007). [doi: 10.1109/tcbb.2007.1012].

[26] H. J. Rong, Y. S. Ong, A. H. Tan and Z. Zhu, “A fast pruned-extreme learning machine for classification problem,” Neurocomputing, 72(1-3), 359-366, (2008). [doi:10.1016/j.neucom.2008.01.005].

[27] A. H. Nizar, Z. Y. Dong and Y. Wang, "Power utility nontechnical loss analysis with extreme learning machine method,” IEEE Trans. Power Syst., 23(3), 946-955, (2008) [doi: 10.1109/TPWRS.2008.2008251].

[28] G. B. Huang, Q. Y. Zhu and C. K. Siew, "Extreme learning machine: theory and applications,” Neurocomputing, 70(1-3), 489-501, (2006). [doi:10.1016/j.neucom.2005.12.126].

[29] C. Rao and S. Mitra, "Generalized Inverse of the Matrix and Its Applications," J. Wiley, New York, (1971).

[30] J. Baglama and L. Reichel, "Restarted block Lanczos bidiagonalization methods," Numerical Algorithms, 43(3), 251-272, (2006). 
[31] J. Sklansky, “Image segmentation and feature extraction,” IEEE Trans. Syst. Man Cybern. , 8(4), 237-247, (1978) [doi: 10.1109/TSMC.1978.4309944].

[32] R. Jobanputra and D. A. Clausi, "Preserving boundaries for image texture segmentation using grey level co-occurring probabilities,” Pattern Recognit., 39(2), 234-245, (2006) [doi:10.1016/j.patcog.2005.07.010].

[33] R. G. Congalton, “A review of assessing the accuracy of classifications of remotely sensed data,” Remote Sens. Environ., 37(1), 35-46, (1991). [doi:10.1016/0034-4257(91)90048-B].

[34] R. A. Monserud and R. Leemans, "Comparing global vegetation maps with the Kappa statistic,” Ecol. Modell., 62(4), 275-293, (1992). [doi:10.1016/0304-3800(92)90003-W].

[35] P. D. Heermann and N. Khazenie, “Classification of multispectral remote sensing data using a back-propagation neural network,” IEEE Trans. Geosci. Remote Sens., 30(1), 81-88, (1992).

[36] J. Campbell, Introduction to Remote Sensing, The Guilford Press, (2007).

[37] P. Domingos and M. Pazzani, "On the Optimality of the Simple Bayesian Classifier under Zero-One Loss,” Machine Learning, 29(2), 103-130, (1997). [doi: 10.1023/A:1007413511361].

[38] F. Melgani and L. Bruzzone, "Classification of hyperspectral remote sensing images with support vector machines,” IEEE Trans. Geosci. Remote Sens., 42(8), 1778-1790, (2004). [doi: 10.1109/TGRS.2004.831865].

[39] "MATLAB technical documentation". Retrieved 2010-06-07. Mathworks.com. http://www.mathworks.com/access/helpdesk/help/techdoc/matlab.html. 